\title{
IDOSOS E O USO DE TECNOLOGIAS ASSISTIVAS EM CASA: UMA REVISÃO SISTEMÁTICA DE LITERATURA
}

\section{ELDERLY AND THE USE OF ASSISTIVE TECHNOLOGIES AT HOME: A LITERATURE SYSTEMATIC REVIEW}

\author{
Hélter Melo, Bach \\ helter.melo@ufpe.br \\ Walter Correia ${ }^{1}$, D.Sc. \\ walter.franklin@ufpe.br \\ Fábio Campos ${ }^{1}$, D.Sc. \\ fabio.ccampos@ufpe.br
}

\begin{abstract}
${ }^{1}$ Laboratório de Concepção e Análise de Artefatos Inteligentes, Universidade Federal de Pernambuco, Recife, Brasil
envelhecimento, tecnologias assistivas, casa, design, segurança e usabilidade

As Tecnologias Assistivas (TAs) possuem grande potencial de auxiliar o envelhecimento saudável em casa e seus campos de atuação atingem diversos setores, exigindo a demanda progressiva de melhoramentos e novas soluções. Nesse sentido, este artigo objetiva compreender o direcionamento dos estudos relacionados ao desenvolvimento de TAs com foco em idosos no ambiente doméstico, nos últimos 5 anos. Para isso, uma revisão sistemática de literatura em quatro etapas foi realizada segundo as recomendações PRISMA. Scopus, SciELO e Google Scholar foram utilizados como bases de dados, com termos pré-definidos em publicações em Inglês e Português, no período de 2015 a 2019. Foram elegidos 36 estudos para a revisão, observando-se a presença do desenvolvimento de tecnologias relativas a artefatos físicos e digitais, ambientes de vida assistida, produtos vestíveis e imersivos, além da implementação de serviços para o ambiente doméstico. Da análise dos estudos, percebeu-se a valorização de questões relativas à privacidade, custo e personalização nos projetos, além do surgimento de novos contextos de ação quando comparado aos trabalhos relacionados anteriores.
\end{abstract}

aging, assistive technologies, home, design, security and usability Assistive Technologies (ATs) have great potential for healthy aging at home. Its activity reaches several sectors and it focuses on the progressive demand for improvements and new solutions. In this sense, this article aims to understand the studies direction related to ATs development, focusing on the elderly and domestic environment, in the last five years. For this, a systematic literature review was carried out in four stages according to the PRISMA recommendations. Scopus, SciELO and Google Scholar were used as databases, based on pre-defined terms, with publications in English and Portuguese and the period from 2015 to 2019. 36 studies were chosen for this review, observing the development of technologies related to physical and digital artifacts, ambient assisted living, wearable and immersive products, in addition to the services implementation for home environment. From the totality of studies, it was noticeable the valuation of issues related to privacy, cost and customization, in addition to the emergence of new action contexts when compared to the related works. 


\section{Introdução}

Estima-se que, no mundo, o número de idosos ultrapasse o de crianças pela primeira vez na história, fortalecendo as perspectivas para que em 2050 a população mundial com 60 anos ou mais chegue totalizar 2 bilhões de pessoas (WHO, 2014). No Brasil, prevê-se que 1 a cada 4 brasileiros será idoso até 2060 (ALVARENGA e BRITO, 2018). Conforme a mesma pesquisa, o número de idosos com mais de 65 anos superará o de crianças de até 14 anos em 2039.

Essas transformações demográficas constituem uma das mais importantes modificações estruturais verificadas no Brasil e no mundo como um todo. Iniciadas timidamente desde 1940, as mudanças se acentuaram progressivamente e vêm culminando no incremento mais lento do número de jovens, paralelo ao aumento contínuo da população brasileira idosa (IBGE, 2016).

O entendimento do cenário de envelhecimento em vigor é, portanto, crucial para compreender a razão dos idosos estarem usando tecnologia (PEEK et al., 2016). Nesse sentido, as Tecnologias Assistivas (TAs) se tornaram um meio palpável de possibilitar melhor qualidade de vida diária ao idoso.

\subsection{As tecnologias assistivas em casa}

Segundo David (2017, p. 6280), tecnologia assistiva é qualquer ferramenta, dispositivo, software ou sistema que utiliza tecnologia moderna para manter ou aprimorar as capacidades funcionais de indivíduos com necessidades especiais. Vichitvanichphong, Talaei-Khoei e Kerr (2017, p. 3678) definem tecnologias assistivas como produtos tecnológicos que apoiam pessoas no desempenho de suas atividades diárias ou manutenção de suas capacidades de função.

Esses artefatos não só se relacionam com os próprios idosos, mas também são de interesse de profissionais de saúde, gerentes de assistência domiciliar, organizações, designers, fornecedores de tecnologia e fabricantes (PEEK et al., 2016). As TAs e as casas estão diretamente relacionadas a questões como disponibilidade, acessibilidade, aceitabilidade e segurança, a fim de garantir o atendimento eficiente à necessidade que se propõem (GARÇON et al., 2016), tornando-se essencial compreender o que facilita ou impede o uso dessas tecnologias. Não somente para entender questões de aceitação, mas também para indicar como melhorar a implementação e a manutenção delas nos lares de idosos (PEEK et al., 2016).

\subsection{O design e o "aging in place"}

O conceito de aging in place tem se consolidado com a preferência dos idosos em permanecer nos seus próprios lares. Uma crescente variedade de tópicos referentes à habitação, meio ambiente, saúde e tecnologia têm despontado nesse cenário, no qual as TAs se relacionam.

A manutenção da independência e a maior disponibilidade de cuidados não institucionais podem ser indicados como os principais fatores da escolha da própria casa como ponto focal de bem-estar (VASUNILASHORN et al., 2012). As influências externas e o contexto de ambiente ao qual o idoso interage influencia diretamente no uso de tecnologias em âmbitos pessoal, social e físico (PEEK et al., 2016). A ambientação familiar também favorece a diminuição do sentimento de exclusão e facilita o uso e aceitação de TAs (DAVID, 2017).

A literatura acadêmica vem apresentando direcionamentos na relação do ambiente e o uso de TAs, refletindo as preocupações de agentes envolvidos e possibilitando um gama maior de estudos e soluções. Tais ações se destinam a conter os custos crescentes de cuidados e facilitar a manutenção da independência dos idosos

(VASUNILASHORN et al., 2012). 


\subsection{Estudos relacionados}

Estudos anteriores, relacionados aos idosos e o uso de artefatos assistivos, foram identificados na literatura.

Khosravia e Ghapanchia (2016) realizaram uma revisão de literatura com objetivo de identificar (no período entre 2000 e 2015) os problemas enfrentados pelos idosos no cotidiano, as tecnologias já usadas para solucionar esses problemas e as suas eficácias. Os resultados identificaram oito principais problemas de assistência aos idosos, indicando que a robótica, os sensores, a telemedicina e os videogames são exemplos de tecnologias destaques usadas nos estudos.

Outra revisão identificada seguiu a linha de pesquisa do estudo anterior e revisou as principais barreiras à adoção de TAs por idosos (YUSIF, SOAR e HAFEEZ-BAIG, 2016). Os resultados indicaram que há incerteza sobre o tempo apropriado para a intervenção das TAs, além da falta de informações e apoio dos serviços formais.

Também foram identificadas revisões acerca de TAs para idosos com demência (MAIA et al., 2018), da utilização de ferramentas de informática assistivas (BACKONJA et al., 2016) e do uso de ambientes de vida assistida (BYGHOLM e KANSTRUP, 2015).

\subsection{Objetivo}

Tendo em vista o cenário descrito, esta revisão sistemática busca compreender como os estudos em design direcionados ao idoso, que tratam da segurança e usabilidade no ambiente doméstico, têm se delineado nos últimos 5 anos.

Este estudo contribui com a literatura sobre as tecnologias assistivas de diferentes maneiras. Primeiro porque promove a continuidade dos estudos na área do envelhecimento e seu impacto na sociedade atual. Segundo, porque desenvolve uma revisão de tecnologias direcionados ao uso doméstico, foco não identificado em estudos anteriores. Além disso, o período de buscas definido neste artigo é posterior ao dos estudos relacionados identificados. Com esse entendimento, os resultados aqui discutidos atualizam e complementam as indicações fornecidas pelas conclusões dos trabalhos anteriores a esse contexto relacionados.

\section{Metodologia}

A revisão sistemática realizada teve como base uma questão norteadora formulada a partir da ferramenta PICo (População ou Problema, Interesse e Contexto), numa abordagem não-clínica e multidisciplinar (MILLER, 2001). Os idosos foram definidos como população, no interesse de segurança e usabilidade e no contexto do ambiente doméstico.

A estrutura da revisão seguiu as recomendações PRISMA. Essas diretrizes possuem o objetivo de auxiliar a estruturação e o processo da revisão sistemática, consistindo em um checklist com 27 itens e um fluxograma com 4 subetapas: Indicação, Seleção, Elegibilidade e Inclusão (GALVÃO et al., 2015).

Para realização das buscas, foram definidas as bases de dados Scopus, SciELO e Google Acadêmico, escolhidas tendo em vista pesquisas de identificação prévia que atestaram uma diversificação preliminar de artigos com possibilidade de seleção dentre os critérios definidos, a serem explicitados a seguir. Os termos de pesquisa utilizados foram: elderly, design, assistive, home, technology, product, old people, care, artifact, ergonomics, senior, daily, security, aged, safety, consumer, geriatric, usability, helpful, everyday e comfort. Foram incluídos artigos que cumpriram os requisitos estabelecidos pela questão norteadora, com acesso aberto, em português ou inglês e publicados entre 2015 e 2019. 
As 4 etapas de seleção dos artigos identificados ocorreram em 2 fases, assim como prevê o PRISMA (GALVÃO et al., 2015). A primeira fase, ou pré-seleção, consistiu na leitura do título e resumo dos artigos, dos quais foram selecionados 180 estudos para a segunda fase. Essa, por sua vez, caracterizou-se pela leitura integral dos estudos, a análise de risco de viés e a sua consequente inclusão ou exclusão da revisão sistemática (com justificativa).

$\mathrm{Na}$ análise de risco de viés, levou-se em consideração a explanação dos estudos presentes na literatura, o cumprimento da metodologia escolhida pelo estudo e a honestidade na exibição e discussão dos resultados. Cada artigo foi avaliado com uma nota de 1 a 5, (a nota 1 representa o artigo com menor risco de viés, enquanto a 5 representa o maior risco). Artigos com nota menor ou igual a 3 foram considerados elegíveis.

A extração de dados dos artigos selecionados foi feita através de um formulário piloto. Através dele se pôde explicitar os dados referentes a título, autor(es), objetivo, metodologia escolhida pelo estudo, seu PICo, a amostra utilizada e os resultados obtidos. Ademais, as principais informações de cada estudo foram extraídas e organizadas separadamente em um arquivo à parte para análise geral e discussão dos resultados obtidos.

\section{Resultados}

Ao todo, 813 artigos identificados foram analisados, dos quais 36 estudos foram selecionados para esta revisão. Os detalhes do processo da revisão podem ser observados na Figura 1.

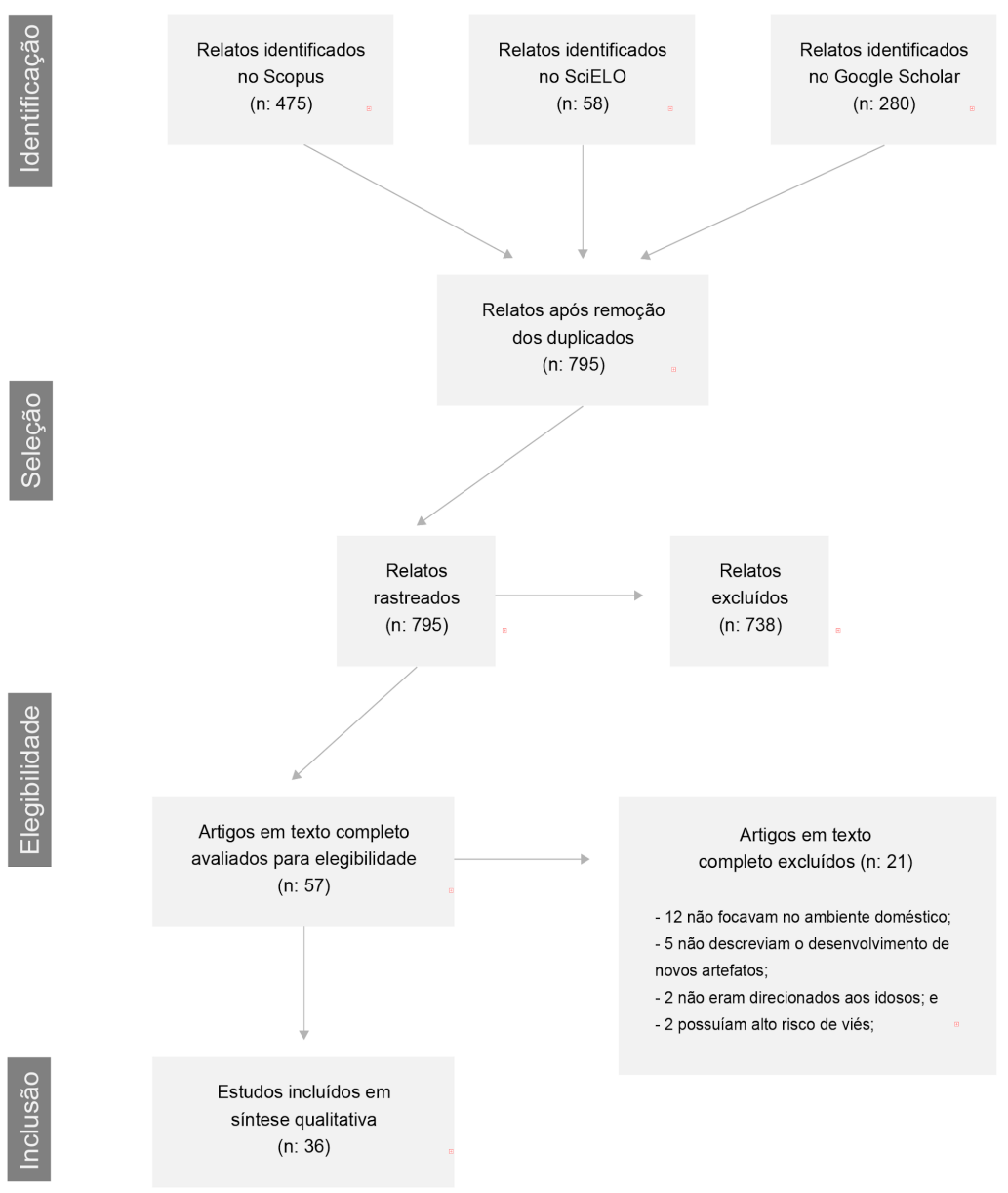

Figura 1. Fluxograma PRISMA da seleção da revisão sistemática. Fonte: Autores. 


\subsection{Ambientes domiciliares (AALs) e robôs assistivos}

A necessidade de assistência na vida diária, advinda com o envelhecimento, perpassa pelas áreas de segurança, integração social, saúde e mobilidade (DO et al., 2018; LOPEZ-GUEDE et al., 2015). Num curso de mudanças demográficas em que as pessoas ficam cada vez mais velhas, os Ambientes de Vida Assistida (AALs, do inglês) apoiam o processo de um envelhecimento mais seguro e confortável (SPINSANTE e GAMBI, 2015). Segundo Do et al. (2018, p. 75), um ambiente doméstico inteligente é definido como uma aplicação onipresente capaz de fornecer aos usuários serviços de assistência com reconhecimento de contexto e automação residencial. A casa passa a interagir com as pessoas e a executar seus comandos, assim como age de forma independente, em casos de emergência.

A característica essencial dos AALs é criar objetos de alta tecnologia que objetivam não ser invasivos, mas simples e fáceis de usar (FRONTONI et al., 2017). Ainda segundo Frontoni et al. (2017, p. 3), os aplicativos domésticos e de construção inteligentes são particularmente importantes para o cenário da Internet das Coisas (IoT), pois representam o vínculo entre o indivíduo (cidadão, consumidor) e as camadas subjacentes (cidades, suas estruturas).

Quanto ao desenvolvimento de robôs assistivos, o mercado já aponta a disposição de alguns modelos, utilizados para ações gerais ou especificidades (DO et al., 2018). Segundo Koceska et al. (2019, p. 1), as principais categorias de robôs de serviço pessoal incluem aqueles para tarefas domésticas, entretenimento, assistividade, transporte, segurança e vigilância.

Mais relacionados ao idoso, os robôs de serviço são ferramentas e companheiros sociais que colaboram e interagem nas tarefas diárias (DO et al., 2018). Fischinger et al. (2016, p. 61) classificam os robôs assistivos como robôs sociais, de serviço doméstico e de sistemas de telepresença. Robôs de companhia social objetivam diminuir a sensação de solidão e geralmente são projetados de maneira a substituir animais de estimação reais. Os de serviço doméstico são aqueles para a realização de tarefas domésticas, a fim de permitir um envelhecimento mais autônomo. Por último, os sistemas de telepresença para idosos se destinam a possibilitar assistência médica e social para a comunicação com pais, enfermeiros, médicos e pacientes.

Recentemente, e evidenciado por artigos incluídos nesta revisão, a integração de sistemas de AALs e robôs assistivos têm proporcionado uma melhor precisão e ampliação nos serviços prestados por esses dispositivos. Como confirmam Do et al. (2018, p. 75), os robôs de serviço doméstico podem utilizar as redes de sensores residenciais inteligentes assim como seus próprios sensores, o que lhes permite ajudar melhor os idosos e colaborar com os cuidadores remotos. Segundo os autores, as tecnologias presentes em residências inteligentes podem ser usadas para melhorar os robôs móveis em termos de desempenho e segurança, reduzindo custos.

Do total de artigos incluídos nesta revisão, 10 estudos se referem ao desenvolvimento de AALs, 6 artigos descrevem a criação e análise de robôs de assistência e outros 2 estudos tratam de sistemas que integram robôs e AALs. A Tabela 1 sumariza as suas informações quanto à descrição, objetivo, amostra e principais resultados obtidos.

\section{Estudo (Local)}

MONTERIÙ et al. 2018 (Itália)
Descrição / Objetivo

Rede integrada de sensores para monitorar o usuário e o ambiente, a fim de obter informações sobre o comportamento do usuário, seu estado de saúde, condição social, etc. Inclui sensores biomédicos, vestíveis e discretos.

\section{Amostra \\ Resultados}

13 idosos que Os sensores utilizados tiveram fácil moram em 8 aceitação e uso pelos usuários, embora apartamentos. tenha sido necessário um treinamento anterior. Ainda é preciso usar de uma arquitetura escalável para armazenamento, análise e compartilhamento de todos os dados da casa. 
BUONCOMPAGNI et al., 2017 (Itália)

RANTZ et al., 2015 (Estados Unidos)
Kit de dispositivo modular para ajudar idosos e pessoas com necessidades especiais tanto na fase póshospitalização quanto quando é necessário ter um serviço de atendimento de médio ou longo prazo em casa.

FATTAH et al., 2017 (Coreia do Sul)

FRONTONI et al. 2017 (Itália)

SPINSANTE GAMBI, 2015 (Itália)

DEMIR et al., 2017 (Turquia) MOŠMONDOR; HULJENIĆ, 2019 (Croácia)

LUO et al., 2018 (Estados Unidos)

LOPEZ-GUEDE et al., 2015 (Espanha) pessoal em ambientes domésticos orientado para idosos que vivem independentemente. Inclui uma máquina de suporte à decisão para prevenção automática de riscos, respondendo a situações em tempo real.

Não explicitada. Com o uso do Kit, os usuários puderam exibir um bom grau de autonomia. Usando outros tipos de vestuário, o sistema pode ser estendido a outros perfis de pessoas com distúrbios cognitivos mais graves.

Sistema de sensor interno discreto que monitora continuamente os idosos quanto ao risco, prevenção e detecção de quedas.

19 idosos que moram em 16 apartamentos.

Não explicitada. sua rotina de medicamentos com a ajuda de lembretes e alertas por meio de produtos da $l o T$, incluindo um relógio de pulso, smartphone e eletrodomésticos como luzes e alto-falantes.

Ambiente doméstico autônomo e automatizado, direcionado a idosos e pessoas com deficiências físicas e motoras.

Aplicativo de software e um dispositivo de hardware para permitir a interação entre idosos com desempenho físico reduzido (ou pessoas com deficiência parcial) em um sistema de automação residencial.

Sistema projetado para detectar e registrar as atividades de um indivíduo idoso que mora sozinho em sua casa.

Serviço inteligente que realiza avaliações reais do usuário (idoso) em um sistema que usa sensores disponíveis no mercado, integrados a um componente de gateway doméstico que transfere dados do sensor para serviços de back-end na nuvem.

Sistema automatizado de monitoramento e análise de padrões nas atividades da vida diária de idosos.
100 agentes envolvidos para o teste de usabilidade da interface do sistema.

20 estudantes.

O Smart Panel, escolhido a partir de testes da tecnologia desenvolvida, oferece interação fácil e confortável. No entanto, ainda são necessários testes adicionais com usuários mais velhos para validar corretamente a interface proposta.

Não explicitada. Os resultados atestaram a eficiência do sistema, embora se acredite que ele será melhor sucedido em ambiente assistivo suportado por uma abordagem baseada em aprendizado de máquina.

13 pessoas, dentre idosos, cuidadores e administradores do sistema.
O sistema foi aceito e bem sucedido, com ressalvas referentes a itens distratores e que podem ser adicionados ao sistema, a fim de uma avaliação mais completa.
Não explicitada.

O modelo alcançou excelentes resultados nas tarefas de classificação de atividade $e$ detecção de atividade temporal contínua, exceto alguns erros identificados.

60 casas de idosos. 
TSUJI et al., 2015 (Japão)

DO et al., 2018

(Estados Unidos)
Sala assistiva equipada com diferentes sensores integrados ao ambiente. A sala é apoiada por um robô humanoide que usa as informações do ambiente para assistir os habitantes da sala.

\begin{abstract}
Casa inteligente integrada a um robô para idosos e uma plataforma do robô para serviço doméstico.
\end{abstract}

Não explicitada.

atividades experimentais realizadas obtiveram ótimos resultados na ação integrada da sala com o robô assistivo. São necessários novos testes com a realização de atividades mais complexas.

12 adultos.

O sistema foi capaz de reconhecer as atividades com uma precisão de mais de $86 \%$, reconhecendo eventos sonoros com uma precisão média de $88 \%$. A detecção de quedas chegou à precisão de $80 \%$, embora possa ser melhorada.

14 idosos e 9 cuidadores. que permite a idosos (ou deficientes) tomar banho de forma independente em casa.

Um robô de telepresença de baixo custo que auxilia os idosos e seus cuidadores profissionais nas atividades cotidianas.
KOCESKA et al., 2019 (Macedônia, Itália, Portugal)
SCHLÖMER, 2018

(Alemanha)

FISCHINGER et al., 2016 (Áustria, Grécia e Suécia)

RANTANEN et al. 2017 (Finlândia)
Robô assistivo com o objetivo de ação em três aspectos: diminuição da solidão, apoio nas tarefas domésticas e assistência médica e social por meio de comunicação remota.
26 idosos e 5 cuidadores.

49 idosos

Fase 1: 17 idosos.

Fase 2: 27 idosos.
Os resultados pontuaram positivamente o artefato. Através das análises da amostra, as suas principais características se referem a autonomia e auto-concepção para os idosos.

O controle compartilhado do robô obteve recursos aprimorados de navegação, reduzindo colisões de obstáculos. Em relação à manipulação do robô, os experimentos mostraram que ele foi altamente eficaz na captura de objetos colocados em locais diferentes.

O sistema robótico pôde executar suas tarefas principais de maneira satisfatória para o grupo-alvo. Todos os participantes foram capazes de executar todas as tarefas em conjunto com o robô e o avaliaram como utilizável e aceitável.

O uso acidental de medicamentos e doses perdidas foi minimizado, com adesão potencial melhorada. A falta de entrega de sachê de medicamentos diminuiu $63 \%$ da Fase I para a Fase II. No entanto, existem limitações quanto ao som do robô e o tamanho da amostra utilizada.

Tabela 1. Sumarização das informações dos artigos selecionados referentes ao desenvolvimento de AALs e robôs assistivos. Fonte: Autores.

\subsection{Tecnologias vestíveis}

Os serviços de monitoramento vestíveis têm o objetivo de entender as atitudes, atividades e contextos aos quais o usuário está submetido, utilizando dados coletados da rede de sensores corporais a ele conectado (DO et al., 2018). De acordo com Biagetti et al. (2018, p. 64), os sensores vestíveis estão revolucionando nossa vida, interação social e atividades da mesma maneira que os PCs fizeram nas últimas décadas.

O uso das tecnologias vestíveis é principalmente direcionado à medição das alterações nos sinais vitais do usuário e detecção de emoções ou status humano. Suas aplicações podem ser categorizadas para uso médico ou não. As aplicações não médicas mais utilizadas incluem detecção de movimento e gestos, reconhecimento cognitivo e emocional e assistência médica (NEGRA; JEMILI; BELGHITH, 2016). 
A integração das tecnologias vestíveis, em geral dotadas de sensores corporais, às informações adicionais como temperatura ambiente, pressão ambiente, luz ou umidade geram um sistema mais completo e preciso como assistência à pessoa idosa (NEGRA; JEMILI; BELGHITH, 2016).

Foram selecionados 6 artigos que se referem especialmente a este tipo de tecnologia, sumarizados na Tabela 2. Além deles, alguns estudos referentes aos AALs, e anteriormente explicitados, possuem tecnologias vestíveis integradas e descritas por eles.

\section{Estudo (Local)}

NORMANN et al. 2017 (Alemanha)

et al., 2016

(China)

VAN OMMEREN et al., 2019 (Países Baixos)

NEGRA; JEMILI; BELGHITH, 2016 (Tunísia)

BIAGETTI et al., 2018 (Espanha)

CAJAMARCA et al. 2018 (Chile)
Eletrodo de eletrocardiografia (ECG) flexível à base de grafeno somado a um sistema portátil de medição de ECG sem

fio.

Baterias têxteis, estruturadas em 2D e $3 \mathrm{D}$, que processam material condutor com colocação de fibra sob medida (TFP).

Luva robótica flexível de três dedos e uma unidade de controle que contém um software incorporado. Objetiva controlar a quantidade de força necessária para suportar a força de preensão e as baterias no uso em atividades diárias. "nós" ultraleves.
Não explicitada. O eletrodo proposto ofereceu sensibilidade comparável a dos eletrodos comerciais de $\mathrm{Ag} / \mathrm{AgCl}$. Também há oportunidades para transferir grafeno para uso em outras aplicações biológicas, como detecção eletroencefalográfica (EEG) e eletromiografia (EMG).

8 idosos.

Com a luva, o transporte de objetos leves ocorreu numa velocidade de pico mais baixa, com maior extensão do cotovelo, e a preensão do objeto envolveu uma abertura maior da mão. Nos objetos pesados, a duração da preensão relativa foi mais longa e a duração relativa do transporte com o objeto mais curto, quando comparado sem a luva.

Não explicitada. As aplicações demonstraram boas promessas na melhoria da qualidade de vida das pessoas e no atendimento de muitos requisitos dos idosos, permitindo que eles vivam com maior segurança, saúde e independência. Apesar disso, há pendências relativas à confiabilidade, urgência, segurança e consumo de energia.

Não explicitada.

Alcançou uma precisão geral de $85,7 \%$. Considerando o material de treinamento um tanto escasso usado, pôde ser considerado um bom resultado. adquirir sinais EMG utilizando sensores

Dispositivo vestível de baixo custo para monitorar as atividades diárias por meio da postura espinhal.
30 idosos

(testes de usabilidade).
Os participantes classificaram a usabilidade do dispositivo, além de sua experiência geral, como alta.

Tabela 2. Sumarização das informações dos artigos selecionados referentes ao desenvolvimento de dispositivos vestíveis. Fonte: Autores. 


\subsection{Tecnologias assistivas para especificidades de saúde}

A especificidade de doenças que acometem pessoas idosas pode gerar a necessidade de estudos direcionados mais profundos e, consequentemente, o desenvolvimento de tecnologias assistivas ad hoc. Dos artigos selecionados, 3 estudos se enquadram nessa temática.

Um estudo de caso realizado com 16 idosos em Betim (Minas Gerais) teve o objetivo de descrever o desenvolvimento de tecnologias assistivas para facilitar a higiene bucal diária em idosos com comprometimentos persistentes relacionados à hanseníase (FERREIRA et al., 2018). O estudo teve uma abordagem personalizada para cada caso, levando em consideração o histórico médico, o comprometimento físico e o ambiente de vida do idoso. Seis meses após a intervenção (o fornecimento de tecnologias assistivas físicas personalizadas), os participantes foram avaliados sobre o uso dos dispositivos. Os resultados indicaram que as tecnologias facilitaram a higiene bucal na maioria dos idosos.

Scott et al. (2018) realizaram uma revisão de literatura sobre TAs desenvolvidas com direcionamento à sarcopenia. Foram encontradas tecnologias relacionadas à mobilidade, monitoramento, sociabilidade e saúde. No entanto, e em geral, todas possuem suas limitações quanto ao uso no cotidiano. Os autores ainda defendem que os médicos devem avaliar as necessidades de TAs em pacientes com sarcopenia.

O terceiro estudo se refere ao acometimento causado pela disfagia, um distúrbio da deglutição que diz respeito à dificuldade (ou impossibilidade) de engolir alimentos ou líquidos (LEE et al., 2017). O estudo piloto, testado com 4 adultos, desenvolveu a ideia de gerar eletrodos conformáveis, ultra-elásticos e vestíveis com a pele para projetar um sistema ergonômico de treinamento de deglutição na reabilitação da disfagia. Toda a área de um eletrodo vestível com a pele é de $1 \mathrm{~cm}^{2}$, e os sinais eletromiográficos analógicos gravados da pele são convertidos, amplificados e transmitidos digitalmente para um receptor externo através de um sistema Bluetooth. Segundo Lee et al. (2017), em comparação com o eletrodo comercial rígido, o sistema semelhante à pele é altamente classificado devido à interface confortável e à aceitação percebida em diferentes ambientes.

\subsection{Dispositivos imersivos}

Um viés estudado, aliado ao melhoramento e complexificação dos robôs assistivos, é a utilização de ambientes imersivos como meio experimental de teste dos dispositivos robóticos. Dessa forma, podem ser analisados questões relativas à disposição de robôs e a sua relação com o espaço, por exemplo.

O estudo realizado por Kim et al. (2017) age com esse foco, sugerindo uma diretriz que ajuda a entender as barreiras para a implementação de um robô em ambiente doméstico. Como afirmam os autores, a operação e a mobilidade do robô auxiliar requerem e ocupam espaço, portanto as especificações de um robô devem considerar o seu ambiente de vida (tamanho do espaço, design de interiores, etc.). Um robô de tamanho humano pode não ser útil em um apartamento menor. Em vez disso, um robô auxiliar pequeno e montado na parede pode fornecer a funcionalidade sem sacrificar a utilização do espaço para a vida diária.

Como auxílio nessas atividades diárias, a utilização de realidade aumentada e projeções interativas também podem ser itens complementares na promoção de uma melhor qualidade de vida. No estudo realizado por Rohrbach et al. (2019), aplicativos de realidade aumentada foram usados por 10 idosos como auxiliares na realização de tarefas diárias (produção de chá), fornecendo o passo-a-passo necessário para finalizar a atividade. Consoante a isso, Hyry et al. (2017) desenvolveram um estudo de uso de um sistema ProCam de projeção interativa, fornecendo ao idoso oito opções assistivas para uso cotidiano que pode ser controlado de a partir de um anel vestível ligado ao sistema. Ambos os estudos obtiveram amplos resultados positivos, embora questões relativas ao conforto do uso de tecnologias vestíveis, como os óculos de realidade virtual, foram levantadas. 


\subsection{Jogos assistivos}

Os jogos sérios são considerados uma alternativa de permitir que idosos mantenham boas condições de saúde na prática exercícios cognitivos e físicos (MOCANU et al., 2017). Mehra et al. (2019) criaram o aplicativo "VITAMINA", projetado para ajudar os idosos a fazerem exercícios em casa. O artefato foi avaliado por 15 idosos, e apesar de que as tarefas puderam ser concluídas com sucesso pela maioria dos participantes, seu desempenho variou bastante nas diferentes tarefas realizadas. Globalmente, no entanto, os participantes foram positivos: 31 observações positivas foram feitas contra 10 observações negativas.

Mocanu et al. (2017) desenvolveram a plataforma "Mobile@Old", que combina o monitoramento de saúde com um jogo para estimular exercícios físicos adaptados a pessoas idosas. O componente de monitoramento de saúde reúne dados médicos de diferentes sensores, processa todos os dados coletados e gera lembretes em casos de emergência. Hong, Kong e Yoon (2018), por outro lado, criaram um programa de exercícios de telepresença que inclui três sessões por semana, realizadas em dias não consecutivos. Cada sessão consiste numa atividade de aquecimento, atividade principal e atividade de relaxamento. Ambos os artefatos obtiveram escores positivos de avaliação das amostras.

No contexto cognitivo, o protótipo "Brain Win" foi projetado para envolver quatro tipos de tarefas cognitivas (discriminação, visuomotora, resposta e cálculo) envolvidas em seis contextos de jogos relacionados à experiência de vida dos idosos (LU; LIN; YUEH, 2017). Os botões e ícones utilizados possuem uma aparência esqueumórfica para que usuários possam conectá-los à sua experiência de vida. De acordo com os resultados provenientes dos cinco idosos que o avaliaram, o jogo é satisfatório e aceitável.

\subsection{Artefatos eletrônicos físicos}

Em menor volume se constatou a presença de estudos que desenvolveram artefatos propriamente físicos, envolvendo uma parte em hardware e outra em software.

Tseng e Hsu (2019) desenvolveram uma cadeira equipada com sensores por meio do qual o suporte emocional intergeracional dos usuários pôde melhorar significativamente. Segundo os autores, após a análise com 6 usuários ( 3 idosos e 3 filhos), a coleta de informações sobre a vida diária dos idosos permitiu que membros remotos da família compreendessem a vida e a saúde um do outro, o que é uma função importante para melhorar as relações intergeracionais.

LUO et al. (2019) criaram um sistema de serviço de coleta da força de preensão. O design do produto final orientou melhor o monitoramento e o gerenciamento da saúde dos idosos, fortalecendo a prevenção de riscos à saúde e melhorando a experiência do usuário em comparação com os produtos existentes. Para a realização do estudo, 60 idosos foram entrevistados e outros 6 participaram do teste de usabilidade (LUO et al., 2019).

\subsection{Demais tecnologias assistivas}

Uma proposta guarda-roupa conceito adaptado para idosos é exposto por Gu e Zeng (2019), através da análise dos problemas existentes no uso do guarda-roupa convencional. O estudo foi realizado através da observação de 13 idosos e entrevistas realizadas a 381 pessoas. As mudanças focaram nos itens de design do tamanho, altura, profundidade, largura, espaço funcional, posição de armazenamento da colcha e aplicação de um trilho de roupa elevável (GU e ZENG, 2019).

Por fim, outro estudo envolveu 33 stakeholders na criação de um sistema que une idosos a prestadores de serviços próximos, no intuito de facilitar a realização de atividades de vida diária. A interface principal do usuário inclui uma tela inicial que mostra um painel de controle, categorizando as funções do sistema em três principais: fornecedores de serviços, usuários de serviços e ferramentas. O sistema foi classificado por $87 \%$ dos sujeitos como "muito útil" ou "útil" (BOND et al., 2015).

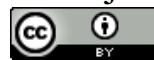




\section{Discussão}

Os estudos resultantes desta revisão de literatura evidenciaram a continuidade de esforços em pesquisa e desenvolvimento de artefatos perante o progressivo envelhecimento da população mundial. O volume de relatos encontrados a respeito do desenvolvimento de sistemas digitais e da prestação de serviços foi mais proeminente em comparação ao desenvolvimento de tecnologias proeminentemente físicas. Essas, em geral, foram utilizadas como artefatos complementares de um sistema, e não totalitárias da solução proposta. Também se observou uma carência geral em relação a estudos próprios da área de design. A maioria dos artigos selecionados são provenientes de áreas afins, como relativas às engenharias e gerontecnologia.

Em geral, a relação do idoso com o seu próprio lar tem ganhando força e sido representado nos estudos. Como exposto por Tseng e Hsu (2019), as interações profundas entre idosos e seus filhos geralmente ocorrem em residências particulares. Fattah et al. (2017) complementam explicitando a necessidade de se considerar um suporte tecnológico que permita que as pessoas idosas morem em suas próprias casas enquanto puderem cuidar de si mesmas. Assistencializar com segurança a relação já estabelecida entre os idosos e seus ambientes é, portanto, valorizar sua independência.

Quando se fala em convivência com sistemas, uma das barreiras quanto à implementação de novos artefatos é a sua própria aceitação pelo idoso. Segundo Hyry et al. (2017), os idosos confiam em modelos mentais existentes de experiências passadas. Isso também se aplica ao uso da tecnologia, mas a falta de experiência com ela cria obstáculos para a interação com o usuário. A não aceitação ou a incapacidade inicial de usar um novo sistema também é discutido por Monteriù et al. (2018). Por isso, enquanto os idosos ainda se sentirem frustrados perante à tecnologia, profissionais devem continuar preocupados e em busca de soluções que possam ajudar (GRGURIĆ; MOŠMONDOR; HULJENIĆ, 2019).

A principal característica presente nos estudos foi a integração de diversas tecnologias num sistema geral que as compõem, coordenando-as e as analisando num contexto em que o idoso é o protagonista. A tendência de integração, portanto, parece se evidenciar como tendência facilitadora na gestão de tecnologias assistivas. Segundo Frontoni et al. (2017), há uma evidência crescente de que a acessibilidade e a usabilidade em um ambiente inteligente por usuários não podem ser obtidas com soluções ad hoc. Por outro lado, Grgurić, Mošmondor e Huljenić (2019) afirmam que se torna mais evidente que sistemas verticais fortemente integrados têm seus benefícios, mas às vezes desvantagens ainda maiores.

\subsection{Personalização}

Nem todos os usuários são iguais e o segmento de pessoas idosas é muito heterogêneo. É importante que sistemas desenvolvidos atendaa a uma necessidade específica do usuário e que o valor que ele ofereça seja mais significativo do que a desvantagem que possa ocorrer (GRGURIĆ; MOŠMONDOR; HULJENIĆ, 2019). De acordo com Lopez-Guede et al. (2015), a situação de um usuário é derivada de seu contexto pessoal, mas o contexto é derivado da agregação de todas as situações dele e da situação do seu ambiente.

Seguindo essa linha, foram observadas evidências sobre personalização nos estudos realizados por Ferreira et al. (2018), Fischinger et al. (2016), Monteriù et al. (2018) e Hyry et al. (2017).

\subsection{Precisão}

Com o melhoramento contínuo de sistemas anteriores, a análise em busca de prover maior precisão dos artefatos foi outro quesito identificado nos estudos, apresentado em Demir et al. (2017), Rantz et al. (2015) e Scott et al. (2018). Os últimos autores afirmam que um dos grandes problemas nos projetos de AALs é justamente a falta de precisão no reconhecimento de comportamento, porque o sujeito monitorado pode executar movimentos contextuais de várias maneiras diferentes. 
Outro problema derivado na busca de precisão é conseguir, em sistemas AAL, definir o que é comportamento "normal" e o limite usado para identificar uma situação anômala. A noção de "normal" é dinâmica e pode se alterar ao longo do tempo e do contexto. As anomalias podem ser identificadas em diferentes escalas temporais, como eventos únicos, dias, semanas ou meses (GRGURIĆ; MOŠMONDOR; HULJENIĆ, 2019).

\subsection{Custo}

Os estudos selecionados foram, em boa parte, realizados em países considerados desenvolvidos. A razão pode estar contida no fato desses países estarem se tornando velhos mais rapidamente. Paradoxalmente, no entanto, a maioria dos idosos se encontra em países menos desenvolvidos, que também estão apresentando um envelhecimento populacional relativamente rápido (BUONCOMPAGNI et al., 2017). Projeções populacionais reafirmam esse raciocínio, estimando que cerca de $80 \%$ dos idosos viverão em países de baixa e média renda em 2050 (WHO, 2014).

Mesmo devido à queda global dos preços proveniente da concorrência gerada pelo desenvolvimento de novos artefatos (SCOTT et al., 2018), o custo desses dispositivos ainda é muito alto. A oportunidade de uso das tecnologias assistivas, portanto, ainda é desigual e afeta diretamente o bem estar dos países menos desenvolvidos.

Diante dessa realidade, Fischinger et al. (2016) estipularam um custo máximo para produção do robô assistivo do seu estudo. Koceska et al. (2019), Tsuji et al. (2015) e Negra, Jemili e Belghith, (2016) também demonstraram preocupação relativa a essa questão em seus estudos.

\subsection{Privacidade}

Num contexto mundial em que os dados possuem muito valor, a preocupação com a privacidade dos dados utilizados nas TAs foi (e deve) estar em pauta para ser discutido. Segundo Scott et al. (2018), em aplicação de AALs, onde os sensores costumam ser usados para recuperar informações sobre a pessoa assistida, é muito importante considerar questões de privacidade e ética, e o sistema de visão deve ser modificado em relação à privacidade.

Quanto à mudança no modo de visão das câmeras, vários estudos optaram por esse modelo (TSUJI et al., 2015; DO et al., 2018; RANTZ et al., 2015; GRGURIĆ; MOŠMONDOR; HULJENIĆ, 2019; LUO et al., 2018).

Em relação aos robôs assistivos, Klein e Schlömer (2018) também afirmam que a maneira como os instrumentos de avaliação ética são utilizados pode contribuir para uma maior abertura de possibilidades de desenvolver respostas sobre questões como, por exemplo, o que é um bom atendimento e qual o impacto que os robôs cuidadores têm sobre o conceito de cuidado, ética e sociedade. Fischinger et al. (2016) reafirmam a atenção em relação à privacidade em seu estudo, estipulando que o robô desenvolvido não deve seguir o usuário todo o tempo, em locais como banheiros.

\subsection{Limitações dos estudos}

Embora observado a busca por melhoramento dos sistemas e tecnologias entre si, inclusive entre os anos desta própria revisão sistemática, há limitações que ainda devem ser estudadas e aperfeiçoadas em trabalhos futuros.

Em AALs, foram observados problemas em relação à precisão dos resultados em contextos em que há mais de uma pessoa dentro de casa (MONTERIÙ et al., 2018; RANTZ et al., 2015). Além disso, como nesses 
sistemas há a necessidade de processamento de muitos dados em tempo real, problemas podem surgir (FRONTONI et al., 2017), especialmente porque a NFC (tecnologia que utilizada na maioria dos AALs) não foi inicialmente projetada para transferir grandes quantidades de dados simultaneamente (SPINSANTE e GAMBI, 2015).

As tecnologias vestíveis, por sua vez, embora possuam grandes possibilidades de utilização, necessitam de ajustes e novas soluções. A manutenção e a confiabilidade no recolhimento de dados em atividades com grandes movimentos são questões importantes. Além disso, o uso de tecnologias assistivas acopladas a peças de roupa fixas acaba limitando a escolha de roupas pelo idoso, o que padroniza as suas escolhas pessoais e não respeita sua integridade individual.

Também não foi identificado uma preocupação formal em parte dos estudos na análise das tecnologias desenvolvidas sob o viés usuário, focando em questões como usabilidade e interação. O que pode explicar isso é o fato de haver poucos estudos focados em design, reafirmando a necessidade do profissional de design no processo de desenvolvimento desses artefatos.

\subsection{Limitações da revisão sistemática}

As limitações da revisão de literatura realizada estão na especificidade em relação ao idioma, a seleção de apenas estudos abertos e às bases de dados pesquisados, definidos nos critérios de inclusão da revisão. Não foram incluídas outras bases de dados com o intuito do cumprimento correto do cronograma da realização da revisão e da presença de resultados obtidos nas bases a partir dos critérios de seleção antes definidos. Dessa forma, há a possibilidade de existirem estudos desenvolvidos no período definido (entre 2015 e 2019), publicados em outros idiomas ou bases não consultadas, que não foram abordados.

\section{Conclusão}

O objetivo desta revisão foi entender o direcionamento tomado pelos estudos que relacionam segurança e usabilidade de tecnologias assistivas para idosos. O ambiente doméstico foi escolhido como contexto da revisão pela tendência mundial de valorização do lar como centro de bem estar do idoso, além de que foram encontrados poucos estudos nesse viés, especialmente nos últimos cinco anos. O envelhecimento é uma questão de longo prazo, portanto é essencial uma atualização progressiva e constante dos estudos direcionados a essa área. A diversidade de pessoas idosas e a multilateralidade dos contextos aos quais estão envolvidas, portanto, se tornaram um quesito de peso a ser levado em conta, junto a outros fatores projetuais necessários.

Como trabalhos futuros, é importante analisar a relação entre os avanços dos estudos sobre tecnologias assistivas e a relação com a diminuição dos custos de produção desses artefatos. Além disso, questões relativas à aceitabilidade e implementação das tecnologias também merecem um olhar a respeito.

\section{Referências Bibliográficas}

ALVARENGA, D.; BRITO, C. 1 em cada 4 brasileiros terá mais de 65 anos em 2060, aponta IBGE. Disponível em $<$ https://g1.globo.com/economia/noticia/2018/07/25/1-em-cada-4-brasileiros-tera-mais-de65-anos-em-2060-aponta-ibge.ghtml >. 2018. Acesso em: 30 de outubro de 2019.

BACKONJA, U.; CHI, N.; CHOI, Y.; HALL, A.; LE, T.; KANG, Y.; DEMIRIS, G. Visualization approaches to support healthy aging: A systematic review. Journal of Innovation in Health Informatics, $v$. 23, n. 3, p. 600-610, 2016. 
BIAGETTI, G.; CRIPPA, P.; FALASCHETTI, L.; ORCIONI, S.; TURCHETTI, C. Human activity monitoring system based on wearable sEMG and accelerometer wireless sensor nodes. BioMedical Engineering Online, v. 17, 2018.

BOND, R.; MULVENNA, M.; FINLAY, D.; MARTIN, S. Multi-faceted informatics system for digitising and streamlining the reablement care model. Journal of Biomedical Informatics, v. 56, p. 30-41, 2015.

BUONCOMPAGHI, L.; BRUNO, B.; GIUNI, A.; MASTROGIOVANNIi, F.; ZACCARIA, R. Arianna: towards a new paradigm for assistive technology at home, 2017.

BYGholm, A.; KANSTRUP, A. The Living Challenge of Ambient Assisted Living: a literature review. Proceedings of the 13th Scandinavian Conference on Health Informatics, Tromsø, Norway, 2015.

CAJAMARCA, G.; RODRÍGUEZ, I.; HERSKOVIC, V.; CAMPOS, M.; RIOFRÍO, J. StraightenUp+: Monitoring of posture during daily activities for older persons using wearable sensors. Sensors (Switzerland), v. 18, n. 10, 2018.

DAVID, Pala. Assistive Technology for Seniors and Health-stratified: Definition and Division. International Journal of Applied Engineering Research, v. 12, n 16, p. 6280-6282, 2017.

DEMIR, E.; KÖSEOĞLU, E.; SOKULLU, R.; ŞEKER, B. Smart Home Assistant for Ambient Assisted Living of Elderly People with Dementia. Procedia Computer Science, v. 113, p. 609-614, 2017.

DO, H.; PHAM, M.; SHENG, W.; YANG, D.; LIU, M. RiSH: A robot-integrated smart home for elderly care. Robotics and Autonomous Systems, v. 101, p. 74-92, 2018.

FATTAH, S.; SUNG, N.; AHN, I.; RYU, M.; YUN, J. Building IoT services for aging in place using standard-based IoT platforms and heterogeneous iot products. Sensors (Switzerland), v.17, n. 10, 2017.

FERREIRA, R.; DE FREITAS RIBEIRO, M.; VARGAS-FERREIRA, F.; SAMPAIO, A.; PEREIRA, A.; VARGAS, A.; DE JESUS, R.; FERREIRA, E. Assistive technologies for improving the oral hygiene of leprosy patients residing in a former leprosy colony in Betim, Minas Gerais, Brazil. PLoS ONE, v. 13, n. 7, 2018.

FISCHINGER, D.; EINRAMHOF, P.; PAPOUTSAKIS, K.; WOHLKINGER, W.; MAYER, P.; PANEK, P.; HOFMANN, S.; KOERTNER, T.; WEISS, A.; ARGYROS, A.; VINCZE, M. Hobbit, a care robot supporting independent living at home: First prototype and lessons learned. Robotics and Autonomous Systems, v. 75, p. 60-78, 2016.

FRONTONI, E.; POLLINI, R.; RUSSO, P.; ZINGARETTI, P.; CERRI, G. HDOMO: Smart sensor integration for an active and independent longevity of the elderly. Sensors (Switzerland), v. 17, n. 1, 2017.

GALVÃO, T.; PANSANI, T.; HARRAD, D. Principais itens para relatar Revisões sistemáticas e Metaanálises: A recomendação PRISMA. Epidemiol. Serv. Saúde, Brasília, v. 24, n. 2, 2015.

GARÇON, L.; KHASNABIS, C.; WALKER, L.; NAKATANI, Y.; LAPITAN, J.; BORG, J.; ROSS, A.; BERUMEN, A. Medical and assistive health technology: Meeting the needs of aging populations.

Gerontologist, v. 56, p. S293-S302, 2016.

GRGURIĆ, A.; MOŠMONDOR, M.; HULJENIĆ, D. The smarthabits: An intelligent privacy-aware home care assistance system. Sensors (Switzerland), v. 19, n. 4, 2019. 
GU, C.; ZENG, J. Research on the Design of the Elderly Wardrobe Based on Ergonomics. IOP Conference Series: Materials Science and Engineering, v. 573, 2019.

HONG, J.; KONG, H.; YOON, H. Web-based telepresence exercise program for community-dwelling elderly women with a high risk of falling: Randomized controlled trial. JMIR mHealth and uHealth, v. 6 , n. 5, 2018.

HYRY, J.; KRICHENBAUER, M.; YAMAMOTO, G.; TAKETOMI, T.; SANDOR, C.; KATO, H.; PULLI, $P$. Design of assistive tabletop projector-camera system for the elderly with cognitive and motor skill impairments. ITE Transactions on Media Technology and Applications, v. 5, n. 2, p. 57-66, 2017.

IBGE, Instituto Brasileiro de Geografia e Estatística. Relações entre as Alterações Históricas na Dinâmica Demográfica Brasileira e os Impactos Decorrentes do Processo de Envelhecimento da População. Disponível em < https://www.ibge.gov.br/estatisticas/sociais/populacao/9232-relacoes-entre-as-alteracoes -historicas-na-dinamica-demografica-brasileira-e-os-impactos-decorrentes-do-processo-de-envelhecimentoda-populacao.html?=\&t=sobre $>$. 2016. Acesso em: 30 de outubro de 2019.

KHOSRAVIA, P.; GHAPANCHIA, A. Investigating the effectiveness of technologies applied to assist seniors: A systematic literature review. International Journal of Medical Informatics, v. 85, p. 17-26, 2016.

KIM, J.; KOO, C.; CHA, S. Immersive virtual environment as a promising tool for the elderly-friendly assistive robot design Jeonghwan. ISARC 2017 - Proceedings of the 34th International Symposium on Automation and Robotics in Construction, 2017.

KLEIN, B.; SCHLÖMER, I. A robotic shower system: acceptance and ethical issues. Zeitschrift fur Gerontologie und Geriatrie, v. 51, n. 1, p. 25-31, 2018.

KOCESKA, N.; KOCESKI, S.; ZOBEL, P.; TRAJKOVIK, V.; GARCIA, N. A telemedicine robot system for assisted and independent living. Sensors (Switzerland), v.19, n. 4, 2019.

LEE, Y.; NICHOLLS, B.; LEE, D.; CHEN, Y.; CHUN, Y.; ANG, C.; YEO, W. Soft electronics enabled ergonomic human-computer interaction from swallowing training. Scientific Reports, v. 7, 2017.

LOPEZ-GUEDE, J.; MORENO-FERNANDEZ-DE-LECETA, A.; MARTINEZ-GARCIA, A.; GRAÑA, M. Lynx: Automatic Elderly Behavior Prediction in Home Telecare. BioMed Research International, v. 2015, 2015.

LOU, C.; LI, R.; LI, Z.; LIANG, T.; WEI, Z.; RUN, M.; YAN, X.; LIU, X. Flexible Graphene Electrodes for Prolonged Dynamic ECG Monitoring. Sensors (Basel, Switzerland), v. 16, n. 11, 2016.

LU, M.; LIN, W.; YUEH, H. Development and evaluation of a cognitive training game for older people: A design-based approach. Frontiers in Psychology, v. 8, 2017.

LUO, S.; LI, W.; WEI, M.; HUANG, Y. A preliminary study of an intelligent grip force collector and service system for the elderly. Advances in Mechanical Engineering, v. 11, n. 2, 2019.

LUO, Z.; HSIEH, J.; BALACHANDAR, N.; YEUNG, S.; PUSIOL, G.; LUXENBERG, J.; LI, G.; LI, L.; DOWNING, N.; MILSTEIN, A.; FEI-FEI, L. Computer Vision-Based Descriptive Analytics of Seniors' Daily Activities for Long-Term Health Monitoring. Proceedings of Machine Learning Research, v. 85, 2018. 
MAIA, J.; COUTINHO, J.; SOUSA. C.; BARBOSA. R.; MOTA. F.; MARQUES. M.; SILVA. R.; LIMA. R. Tecnologias assistivas para idosos com demência: revisão sistemática. Acta Paul Enferm, v. 31, n. 6, p. 651658, 2018.

MEHRA, S.; VISSER, B.; CILA, N.; VAN DEN HELDER, J.; ENGELBERT, R.; WEIJS, P.; KRÖSE, B. Supporting older adults in exercising with a tablet: A usability study. Journal of Medical Internet Research, v. 21, n. 2, 2019.

MILLER, Syrene. PICO worksheet and search strategy. US National Center for Dental Hygiene Research, 2001.

MOCANU, I.; SCHPOR, O.; CRAMARIUC, B.; RUSU, L. Mobile@Old: A smart home platform for enhancing the elderly mobility. Advances in Electrical and Computer Engineering, v. 17, n. 4, p.19-26, 2017.

MONTERIÙ, A.; PRIST, M.; FRONTONI, E.; LONGHI, S.; PIETRONI, F.; CASACCIA, S.; SCALISE, L.; CENCI, A.; ROMEO, L.; BERTA, R.; PESCOSOLIDO, L.; ORLANDI, G.; REVEL, G. A smart sensing architecture for domestic monitoring: Methodological approach and experimental validation. Sensors (Switzerland), v. 18, n. 7, 2018.

NEGRA, R.; JEMILI I.; BELGHITH, A. Wireless Body Area Networks: Applications and Technologies. Procedia Computer Science, v. 83, p. 1274-1281, 2016.

NORMANN, M.; GRETHE, T.; ZÖLL, K.; EHRMANN, A.; SCHWARZ-PFEIFFER, A. Development of 2D and 3D structured textile batteries processing conductive material with Tailored Fiber Placement (TFP). IOP Conference Series: Materials Science and Engineering, v. 254, n.7, 2017.

PEEK, S.; LUIJKX, K.; RIJNAARD, M.; NIEBOER, M.; VAN DER VOORT, C.; AARTS, S.; VAN HOOF, J.; VRIJHOEF, H.; WOUTERS, E. Older Adults' Reasons for Using Technology while Aging in Place. Gerontology, v. 62, n. 2, p. 226-237, 2016.

PEEK, S.; WOUTERS, E.; LUIJKX, K.; VRIJHOEF, H. What it Takes to successfully implement technology for aging in place: Focus groups with stakeholders. Journal of Medical Internet Research, v. 18, n. 5, 2016.

RANTANEN, P.; PARKKARI, T.; LEIKOLA, S.; AIRAKSINEN, M.; LYLES, A. An In-home Advanced Robotic System to Manage Elderly Home-care Patients' Medications: A Pilot Safety and Usability Study. Clinical Therapeutics, v. 39, n. 5, p. 1054-1061, 2017.

RANTZ, M.; SKUBIC, M.; ABBOTT, C.; GALAMBOS, C.; POPESCU, M.; KELLER, J.; STONE, E.; BACK, J.; MILLER, S.; PETROSKI, G. Automated In-Home Fall Risk Assessment and Detection Sensor System for Elders. Gerontologist, v. 55, n. 51, p. S78-S87, 2015.

ROHTBACH, N.; GULDE, P.; ARMSTRONG, A.; HARTIG, L.; ABDELZAREG, A.; SCHRÖDER, S.; NEUSE, J.; GRIMMER, T.; DIEHL-SCHMID, J.; HERMSDÖRFER, J. An augmented reality approach for ADL support in Alzheimer's disease: A crossover trial. Journal of NeuroEngineering and Rehabilitation, v. 16, n. 1, 2019.

SCOTT, R.; CALLISAYA, M.; DUQUE, G.; EBELING, P.; SCOTT, D. Assistive technologies to overcome sarcopenia in ageing. Maturitas, v. 112, p. 78-84, 2018.

SPINSANTE, S.; GAMBI, E. NFC-based user interface for smart environments. Advances in HumanComputer Interaction, v. 2015, 2015. 
TSENG, W.; HSU, C. A Smart, Caring, Interactive Chair Designed for Improving Emotional Support and Parent-Child Interactions to Promote Sustainable Relationships Between Elderly and Other Family Members. Sustainability, v. 11, n. 961, 2019.

TSUJI, T.; MOZOS, O.; CHAE, H.; PYO, Y.; KUSAKA, K.; HASEGAWA, T.; MOROOKA, K.; KURAZUME, R. An informationally structured room for robotic assistance. Sensors (Switzerland), v. 15, n. 4, p. 9438-9465, 2015.

VASUNILASHORN, S.; STEINMAN, B.; LIEBIG, P.; PYNOOS, J. Aging in place: Evolution of a research topic whose time has come. Journal of Aging Research, v. 2012, 2012.

VAN OMMEREN, A.; RADDER, B.; KOTTINK, A.; BUURKE, J.; PRANGE-LASONDER G.; RIETMAN, J. Quantifying upper extremity performance with and without assistance of a soft-robotic glove in elderly patients: A kinematic analysis. Journal of Rehabilitation Medicine, v. 51, n. 4, p. 298-306, 2019.

VICHITVANICHPHONG, S.; TALAEI-KHOEI, A.; KERR, D. Elderly's Perception about The Value of Assistive Technologies for their Daily Living: Impacting Factors and Theoretical Support. Proceedings of the 50th Hawaii International Conference on System Sciences (2017), p. 3678-3685, 2017.

WORLD HEALTH ORGANIZATION.. “Ageing well” must be a global priority. Disponível em < https://www.who.int/mediacentre/news/releases/2014/lancetageingseries/en/?fbclid=IwAR2_hi9V5xFgMxH 3nWmQH8Rf_JUFIrjpv54nq2uU-PellwazIEn9LsMVZ-c >. 2014. Acesso em: 27 de outubro de 2019.

YUSIF, S.; SOAR, J.; HAFEEZ-BAIG, A. Older people, assistive technologies, and the barriers to adoption: A systematic review. International Journal of Medical Informatics, v. 94, p. 112-116, 2016.

\section{Agradecimentos}

Este estudo foi realizado através do Programa Institucional de Bolsas de Iniciação Científica (PIBIC), pelo Conselho Nacional de Desenvolvimento Científico e Tecnológico (CNPQ). As etapas de desenvolvimento da revisão sistemática foram realizadas no Laboratório de Concepção e Análise de Artefatos Inteligentes ( $\left.\mathrm{LaCA}^{2} \mathrm{I}\right)$, na Universidade Federal de Pernambuco (UFPE), Recife, Pernambuco, Brasil. 